\title{
A Novel Extended Biometric Approach for Human Character Recognition using Fingerprints
}

\author{
Rohit R Prabhu \\ Research Scholar \\ Department of Computer Science and Engineering \\ Sri Jayachamarajendra College Of Engineering \\ Mysore
}

\author{
C.N. Ravikumar, Ph.D \\ Professor and Head \\ Department of Computer Science and Engineering \\ Sri Jayachamarajendra College Of Engineering \\ Mysore
}

\begin{abstract}
The recent innovations in Biometrics and its allied areas like pattern recognition have made the task of verification and identification, a reality which is savored in many applications like granting Unique Identification Number by the Government to its people. Existing system for verification and identification of a human being is heading towards near perfection. However, these systems cannot declare the psychology of the person. Ancient sages from India had always claimed that every natural or acquired bodily mark encodes its owner's psychology and destiny in their various manuscripts. Palmistry as an abstract science and current systems of pattern recognition can be leveraged in assessing the personality and character of a person which is the basis of our work.
\end{abstract}

\section{General Terms}

Pattern Recognition, Artificial neural networks

\section{Keywords}

Biometrics, Palmistry, Human character analysis, Fingerprints

\section{INTRODUCTION}

It is a popular cliché- Anything can't be trusted without being measured. In everyday life, humans measure many things consciously or sub-consciously. Things that could be touched and sensed pose no problem in measuring. However, measuring intangible features like character of a person is a great challenge. The ancient science of Palmistry has innumerable approaches to measure such intangible characteristics. Although, the entire human body depicts the character of the person, in this work only fingerprint is used to assess personality traits. Palmistry in conjunction with biometrics could be used to realize a system of automatic human character identification which is the endeavor of this work.

\subsection{Biometrics}

The terms "Biometrics" and "Biometry" have been used since early in the 20th century to refer to the field of development of statistical and mathematical methods applicable to data analysis problems in the biological sciences. Recently, these terms have also been used to refer to the emerging field of information technology devoted to automated identification of individuals using biological traits, such as those based on retinal or iris scanning, voice patterns, dynamic signatures, fingerprints, face recognition, or hand measurements, especially for authentication purposes. Thus biometrics can be defined as the science and technology of measuring and statistically analyzing biological data. They are measurable physiological and/or behavioral characteristics that can be utilized to verify the identity of an individual. For a layman, it could be said that biometrics is the science of measuring physical and/or behavioral characteristics that are unique to each individual and they verify that an individual is who he or she claims to be.[1]

Often seen in science-fiction action adventure movies, face pattem matchers and body scanners seem about to emerge as replacements or computer passwords. Thus biometric techniques are of interest in any area where it is important to verify the true identity of an individual. Initially, these techniques were employed primarily in specialist high security applications; however we are now seeing their use and proposed use in a much broader range of public facing situations.

Fingerprint, facial, or other biometric data can also be placed on a smart card and users can present both the smart card and their fingerprints or faces to merchants, banks, or telephones for an extra degree of authentication.[2]

In addition to the applications involving verification and identification, Biometrics is beginning to find usage in wider applications where its help was hardly ever imagined. It is now used as the window to the human brain which helps in analyzing the human behavioral pattern. Biometrics is also finding significant usage in palmistry - art of handreading which is opening up the map of deriving person's past and future.

\subsection{Palmistry}

Palmistry, also known as chiromancy, is a study of the characteristics of the hands, including lines, markings, skin tone and fingernails in order to reveal the past, present and future. These characteristics can reportedly diagnose latent health problems, reveal character traits and guide future actions.[3]

The hand has been ascribed special significance from the Stone Age, illustrated by many cave paintings in Europe and other parts of the Western hemisphere and Bushman paints in Africa. Many ancient cultures including the Egyptians, Greeks and Romans used palmistry to foretell the future. History also records the use of palmistry in ancient India and China.

It is interesting to note, however, that there are many references to palm or hand reading in the Old Testament, Vedic scripts and early Semitic writings. One of the earliest texts on palmistry is understood to be De Philsiognomia by Michael Scotts published in 1477. This described the 
physiognomy of the human body and included a chapter on the human hand. Physiognomy is a pseudo-science with the premise that a person's appearance and visible characteristics reflects character and personality.[4]It is scientifically proved by biological sciences that the shape of the lines, length of the lines, the thickness and color of the lines on the palm is based on the individual thinking pattern.[5]

This paper is organized as follows: in section 2 the classification of Biometrics is presented based on the applications. In section 3 various existing modes and problems associated with human character recognition is described. In section 4 and 5 various fingerprint patterns and usage of fingerprint as a tool to judge human character is explained. Implementation details of the proposed approach is presented in section 6 and in section 7 results of the experimentation is provided. In section 8 some of the possible applications are explored and finally conclusion is provided in section 9 .

\section{CLASSIFICATION OF BIOMETRICS}

In the past, the use of biometric was almost limited to solve significant problems in the field of verification and identification especially in Criminology. Most of the countries are investing heavily in building robust system used for Automated Fingerprint Identification System [AFIS] and palm print recognition system. In fact, Australia currently houses the largest repository of palm prints in the world. The Australian National Automated Fingerprint Identification System (NAFIS) stores over 4.8 million palm prints.[6]

Currently, the trend is changing and Biometrics is finding new home in various applications in psychometric analysis, diagnosis of diseases and future predictions. This has motivated the classification of biometrics in terms of application it is employed rather than the classification based on identification only.[3]

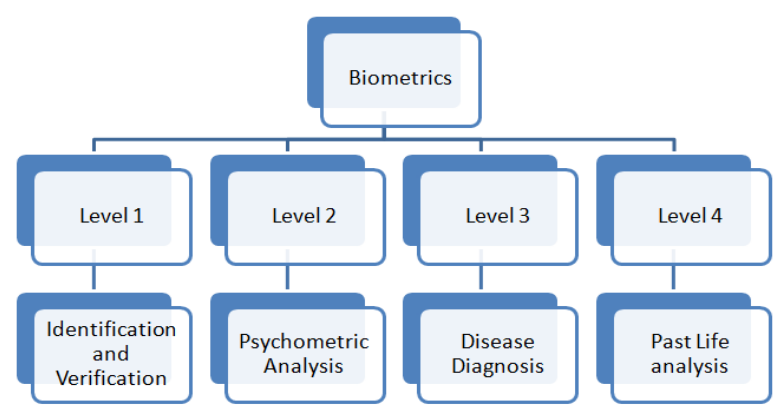

Figure 1: Classification of Biometrics

Looking at the vast majority of application in biometrics it is useful to classify biometrics as depicted in Figure 1 The following sections give a clearer insights about the classifications.

\subsection{Biometric-Level 1}

At this level, depending on the context, a biometric system can be either a verification (authentication) system or an identification system. Verification involves confirming or denying a person's claimed identity while in identification, one has to establish a person's identity. Biometric systems are divided on the basis of the authentication medium used. They are broadly divided as-

$$
\begin{aligned}
& \text { - Face recognition } \\
& \text { - Voice Pattern } \\
& \text { - Vein Pattern } \\
& \text { - Finger Prints } \\
& \text { - Iris Recognition }
\end{aligned}
$$

These methods are used on the basis of the scope of the testing medium, the accuracy required and speed required. Every medium of authentication has its own advantages and shortcomings. With the increased use of computers as vehicles of information technology, it is necessary to restrict unauthorized access to or fraudulent use of sensitive/personal data. Biometric techniques being potentially able to augment this restriction are enjoying a renewed interest.[7]

\subsection{Biometric-Level 2}

Personality is made up of the characteristic patterns of thoughts, feelings and behaviors that make a person unique. Personality is basically understood by three major domains or methods in Indian Philosophy from which Indian Psychology has evolved. They are Pancha Kosha, Pancha Mahabhutas and Trigunas. The concept of trigunas is well treated in [8].

The approach to judge character of human beings using biometrics is discussed in section 3 in depth.

\subsection{Biometric-Level 3}

Biometric-Level 3 deals with the use of biometric techniques to diagnose some of the diseases. The health condition of a person is perceived by the Face and Hands. The Mishio Kushi Theory (Face Reading) contends that a weakness or toxicity in a certain organ or gland can cause acne on certain areas of the body. The radiance on the face represents the credibility of the body health. Similarly Hand interprets the thought impulses of the brain and hence these carvings and also all nerve endings happen to end here. Hence forth the Face and Hand can be made into a continuum of differential areas of face, which in turn represents the health condition of the body.[3]

\subsection{Biometric-Level 4}

Biometric level 4 deals with the use of biometric techniques to decode the soul-level information. According to Hinduism a soul reincarnates again and again on earth till it becomes perfect and reunites with its Source. During this process the soul enters into many bodies, assumes many forms and passes through many births and deaths.

Researchers have suggested that the formation of epidermal ridges on the finger tips of fetus starts from $10^{\text {th }}$ to $16^{\text {th }}$ week of pregnancy and on $16^{\text {th }}$ week the patterns of epidermal ridge get established and don't get changed thereafter.[9] According to yoga philosophy this is about the same time the soul enters the body. There are basically three types of fingerprint patterns: whorls, loops, and arches. Upon examination of the patterns in the fingerprints we can see that it takes a different type of energy to create the pattern of the whorl than it would to create the arch. 
The information encoded in the fingerprints is soul level information. The combination of fingerprints in relation to the fingers that they appear on creates a code. This code forms a map that tells why we have come here in this lifetime.[10]

\section{APPROACHES TO HUMAN CHARACTER RECOGNITION}

THOMAS BABINGTON MACAULAY (1800-1859) British writer and politician once said "The measure of a man's character is what he would do if he knew he never would be found out." To device a method to measure the character of a person, it is important to capture a feature which is neutral to external conditions. Palm gives us a step by step recording of an individual's life from birth to death. Palmistry remains unrelated to worldly events, environment or any external conditions. Hence, Palm in particular, fingers can be used to judge human character.

\subsection{Existing ways to judge characters human}

Nowadays many companies have a round of psychometric test as part of their recruitment drive in order to gauge the character of the candidate when put under various circumstances. These include rapid question fired at the candidate for which the candidate needs to answer spontaneously. The answer gives the good measure of candidate's character to the interview panel.

Psychometric Test involves two major research tasks, namely: (i) the construction of instruments and procedures for measurement; and (ii) the development and refinement of theoretical approaches to measurement. [11]

The major disadvantage of the psychometric test are

- Some candidates will not give their true opinions. They will answer at their ease to get through the paper. It will not support to conduct a successful test to identify their true \& potential judging skills.

- The trait of a character which will be ascribed to a person depends on the situation in which the person's behavior is being observed. Therefore the outcome of psychometric methods may not be accurate, consistent and reliable.[12]

The major reason why psychometric tests fails is that it needs to consider lot of external factors while judging a human being. These external factors make the psychometry unreliable. What if some intrinsic factors of human being are considered which won't change under external conditions? The Biometric-II judges the characteristics of a person taking into account his body type, face, hand, sleeping style, walking gait and such other aspects. Thus the physical appearance comes into greater focus on the grounds that they are constant factors not governed by the intentions of an individual. If these two different approaches are fused, the forthcoming results will be accurate, reliable and consistent.

\subsection{Approaches to human character recognition}

There are approaches which helps in recognizing the personality traits of human beings without the interference of the external influences. Some of these techniques are discussed in the following.

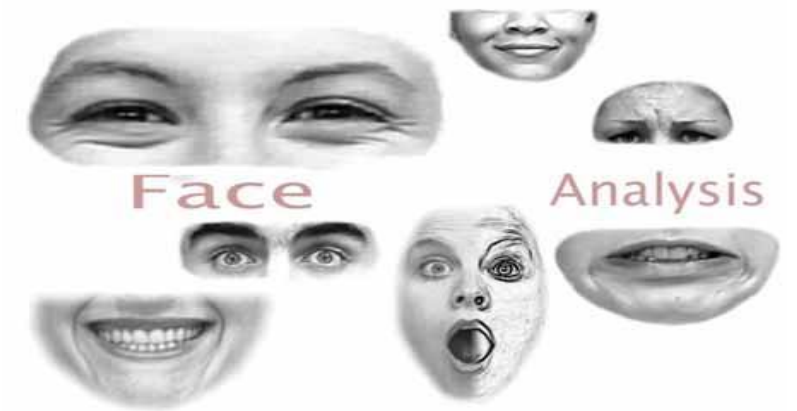

Figure 2: Various facial expressions

\subsubsection{Face Analysis}

Personality analysis based on individual facial features is for the most part subjective. The same holds true for facial analysis. Different people with the same nose, eyes, or mouth, may have totally different personality characteristics. In case of $100 \%$ identical twins the personality and learning ability will be alike.

Shapes of faces such as Round, Square, Rectangular, Triangular and also straight face, concave face, convex face represent laziness, dullness, creativity, humor, fastidiousness, toughness, aggressiveness, offensiveness, sociability, strong physique, honesty, quality for diplomacy and leadership, competence to work as executive and official, anger, being unskilled yet boastful and rough, temperamental cheerfulness and brilliance, hyper-activeness and sensitivity, disturbed married life, equipoise, pessimism, happily disposed optimism, refinement in nature, criminal tendency, self-reliance. Analyzing the character of a human being using various facial features is well described in [3] and [13].

\subsubsection{Palm Analysis}

The hands are a reflection of the mind. By the time the child is born, it brings a map of the life in its hands. This map is a recording of the life till death. Using this map and gift of free will, one can become master of his own destiny. In most schools of palmistry, hand shapes are divided into four or 10 major types, sometimes corresponding to the classical elements or temperaments. Hand shape is believed to indicate character traits corresponding to the type indicated (i.e., a "Fire hand" would exhibit high energy, creativity, short temper, ambition, etc. - all qualities believed to be related to the Classical element of Fire).

Although variations abound, the most common classifications used by modern palmists are

- Square hand

- Spatulate hand

- Pointed hand

- Philosophic hand.

- Psychic or idealist hand

- Conic hand

Their connotations can be found in [14].The number and quality of lines, the mounts in the palm can also be included in the hand shape analysis. 


\subsubsection{Lines}

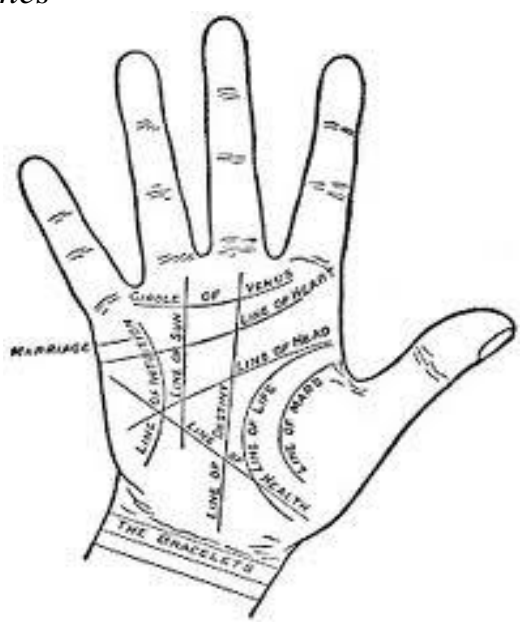

Figure 3: Palm Lines

Aristotle stated that "Lines are not written into the human hand without reason. They emanate from heavenly influences and man's own individuality."[4]

The three lines (also called Major Lines) found on almost all hands, and generally given most weight by palmists include(Figure 3):the Heart Line, the Head Line and the Life Line. The Heart Line represents Love and attraction. It also gives insight on the person's emotional side. The Head Line is used to interpret the person's mindset, it's learning style, intellectualism and thirst of knowledge. The Life Line is believed to represent the person's vitality and vigor, physical health and general well being.

There are other lines called the Minor Lines viz., the Fate Line, the Sun Line, the Girdle of Venus, the Travel Lines, the Marriage Lines which have their own significance. More comprehensive information could be found in [15].

\section{FINGERPRINT AS A WINDOW TO JUDGE HUMAN CHARACTER}

Of the various approaches discussed in section 3 adopted to judge the characteristics of human beings, th i s work is confined to the analysis of character using finger prints because it is through the fingerprints humans connect to the world. The front of the hand is composed of ridges and valleys, and these offer untold possibilities of interpretation, as each one is unique.

A fingerprint in its narrow sense is an impression left by the friction ridges of a human finger. In a wider use of the term, fingerprints are the traces of an impression from the friction ridges of any part of a human or other primate hand.[16]

Unlike the lines of the palm, the lines on the fingertips never change. Each print is unique to a person - like snowflakes, no two fingerprints are alike. No matter how hard people have tried to get rid of their fingerprints - even by burning or cutting the prints away with fire, acid, or a knife - they still grow back the same way. As the child gets older, his or her fingerprints will grow in size but the fingerprint pattern remains the same throughout the life. Hence, the fingerprints have long been used in criminal investigations and for identifying persons

\subsection{Types of Fingerprints}

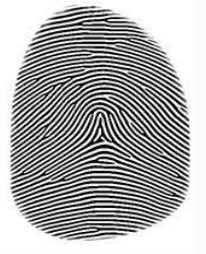

(a)

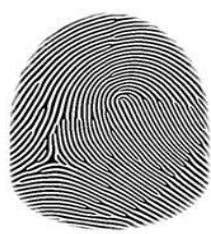

(b)

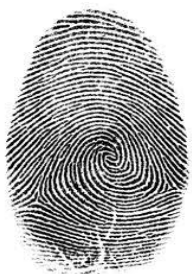

(c)
Figure 4: a) Arch b) Loop c) Whorl

Fingerprints are found in three basic patterns: loops, whorls, and arches (Figure 4). Each of these patterns could be broken down further into subcategories, and each kind of pattern will give valuable insights into person's character. One may have the same pattern of print on each finger, or they may be mixed. If one has the same pattern on all ten fingers, the attributes signified by that pattern will be strongly displayed.

The meaning of the pattern could be judged by its position in the fingerprint. If the center of a pattern (core) is found on the upper part of the fingertip, it represents an intellectual or mental area of concern, but if it is lower, it shows a practical way of dealing with physical matters.

\subsubsection{Loops}

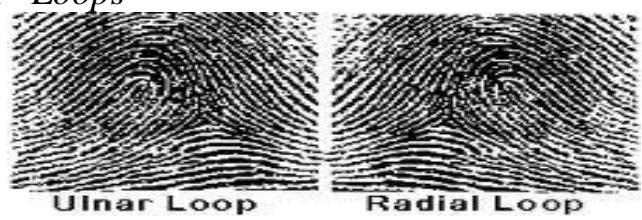

Figure 5: Types of Loops

Loops occur in about $60-70 \%$ of fingerprint patterns encountered. One or more of the ridges enters on either side of the impression, re-curves, touches or crosses the line running from the delta to the core and terminates on or in the direction of the side where the ridge or ridges entered. Each loop pattern has one delta, one core and has a ridge count.

- Radial loops are named after the radius, a bone in the forearm that joins the hand on the same side as the thumb. The flow of the pattern in radial loops runs in the direction of the radius (toward the thumb). Radial loops are not very common and most of the time radial loops will be found on the index fingers.

- Ulnar loops are named after the ulna, a bone in the forearm. The ulna is on the same side as the little finger and the flow of the pattern in a ulnar loop runs in the direction of the ulna (toward the little finger).[17]

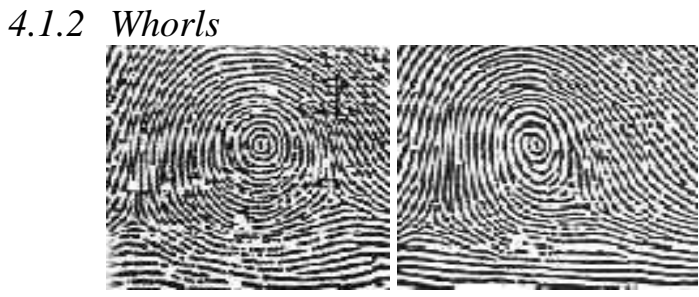

a)

b)

Figure 6: Whorl patterns-a) Concentric b) Spiral 
Whorls are seen in about $25-35 \%$ of fingerprint patterns encountered. In a whorl, some of the ridges make a turn through at least one circuit. Any fingerprint pattern which contains 2 or more deltas will be a whorl pattern. There are basically two types of whorl patterns:

- Concentric whorls consist of one or more ridges which make or tend to make a complete circuit with two deltas, between which an imaginary line is drawn and at least one re-curving ridge within the inner pattern area is cut or touched.

- Spiral whorls consist of at least one recurving ridge or an obstruction at right angles to the line of flow, with two deltas, between which when an imaginary line is drawn, no re-curving ridge within the pattern area is cut or touched. Central pocket loop whorl ridges make one complete circuit which may be spiral, oval, circular or any variant of a circle.

\subsubsection{Arches}

Arches are found in about 5\% of fingerprint patterns encountered. The ridges run from one side to the other of the pattern, making no backward turn. Ordinarily, there is no delta in an arch pattern but where there is a delta, no recurving ridge must intervene between the core and delta points. There are two types of arch patterns: plain arches and tented arches.

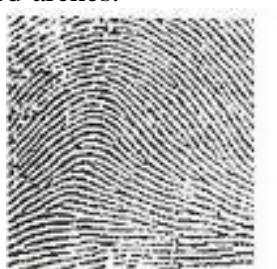

Plain Arch

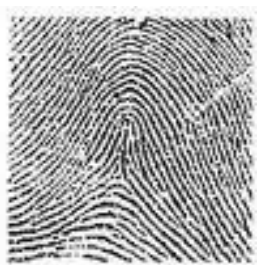

Tented Arch
Figure 7: Arch patterns

- Plain arches have an even flow of ridges from one side to the other side of the pattern, no "significant up thrusts" and the ridges enter on one side of the impression, and flow out the other with a rise or wave in the center

- Tented arches have an angle, an up thrust, or two of the three basic characteristics of the loop. They don't have the same "easy" flow that plain arches do and particularly have "significant up thrusts" in the ridges near the middle that arrange themselves on both sides of a spine or axis towards which the adjoining ridges converge and appear to form tents.[17]

Apart from the above mentioned basic patterns there are other patterns which are formed by the combination of the basic patterns. These patterns are called composite patterns. For e.g.-The Peacock's Eye. More information on these patterns can be found here [18].

\section{JUDGING CHARACTER BASED ON FINGERPRINTS}

Fingerprints and their patterns serve as a valuable tool for assessing the character of a person both in eastern and western palmistry. Each pattern in a fingerprint like appearance of Star, Island, Cross, Triangle, Forks, Chain, Grille and Bar has its own significance depending on the position where they appear. However, in this context only the basic patterns and their connotations with respect to the behavioral aspect of the human beings is considered.

\subsection{Loops}

They are the most common pattern found on fingertips, especially on the thumb. People with a lot of loops get along well with everyone. If one have them, he is very adaptable to new social situations and interested in everything and everyone. He likes to talk to people and find out more about their interests and ideas, and can be a capable or charming leader as well. Also, a loop shows that the person has a very middle-of-the-road ideas in the area represented by the finger that the loop is found on. Also, the location of loops on the various fingers has specific implications. For e.g.: A radial loop on Jupiter finger suggests leadership qualities and bossiness.

As mentioned in section 4.1.1 there are two kinds of loops: Ulnar loops and Radial Loops. People with these loops are still agreeable and enjoyable, but they are more likely to speak up for their own interests and stand out from the crowd than people with ulnar loops.[19], [20]

\subsection{Whorls}

People with whorls, which look like circles, have a strong will and are more controlling and individualistic than others, but they can also be uniquely dynamic and creative - in a "tortured genius" kind of way. They dislike doing anything the way others would like them to and conforming to a set standard. If a person has lot of whorls, this means that he might have many talents, and he is a decent and trustworthy person.

Whorls come in two varieties - spiral and concentric. Spiral whorls are in the shape of spirals, just as the name suggests. If one has these, he is likely adamant about his beliefs and will hold them faithfully throughout his life. He likes to be independent at work and labors best when guided by self.

Whorls that form complete and unspiralled circles, which are known as concentric whorls, are unusual and can be usually found on the Apollo or Jupiter fingers or on the thumb. If the person has concentric whorls, his attitudes are even stronger - he would like to be running the world! Fortunately, that wouldn't be all that bad, because he is a responsible person, trustworthy to the extreme.[20]

\subsection{Arches}

People with arches are hardworking, capable, and commonsensical and they do not procrastinate when it comes to getting the job done - they can be trusted to follow through. They like knowing what they are getting into, and they are private and down to earth, but they can also have a very limited or narrow viewpoint.

Additionally, if the arches are raised(tented arch), a rare condition with the center part sticking up fairly high around a "tent pole," he is little more likely to be enthusiastic and idealistic about your life and to let the feelings conquer the practical side. In fact, if the arch is too high, he may be a little too emotionally sensitive, to the point of being nervous and jumpy, unlike the average down-toearth arched personality. Tented arches are generally found in the fingerprints of emotionally insecure people, while simple arches are typically found in the fingerprints of easygoing people.[20] 


\section{IMPLEMENTATION}

The major challenge of the work is to initially classify the obtained fingerprint into 3 major categories viz., loops, whorls and arches and eventually portray the character based on the fingerprint as per the knowledge gained in section 5. Neural network based pattern recognition principles is used to achieve this task.

\subsection{Proposed Approach}

The method suggested by [21] is used for classification of fingerprints. The various steps in a fingerprint recognition system are shown in Algorithm 1. The first stage is the capturing of the image in which a fingerprint image is obtained from an individual. The captured image of fingerprint is then converted into the binary form. The binary image is then converted into the histogram equivalent. A network is designed which contains neurons in the input layer in the hidden layer as well as in the output layer. The network is trained by applying different number of samples. Once the training phase is completed different samples are applied to the network to check its performance. Finally a test fingerprint is presented to classify the fingerprint into whorls, loops and arches.

Algorithm 1: Human character recognition process
Step 1: Capture the fingerprint
Step 2: Convert the image into binary form
Step 3: Convert the image from binary form to
histogram.
Step 4: Train the neural network
Step 5: Find the pattern (whorl, arch or loop)
Step 6: Display the character exhibited by that pattern.
The goal in this classification problem is to develop an
algorithm which will assign any image, represented by a
vector $x$, to one of the class denoted by $C_{k}$, where, k=1 or
2,so that class $C_{1}$ corresponds to the fingerprint image having
whorls and $C_{2}$ corresponds to the fingerprint image of having
loops. For the sake of simplicity, classification of arch
pattern is not considered. In this work, 48 different
fingerprint images are collected which constitutes the data-
set.

\subsection{Data Preparation}

After finding the histogram equivalent of the fingerprint image of the given binary numbers, these histogram values are taken as input and some random values are assigned as target values to train, test and validate each part individually using feed forward back propagation neural network tool.

Table 1: Sample dataset obtained from 48 fingerprints

01-> Whorl

\begin{tabular}{|c|c|c|c|c|}
\hline Sample No & \multicolumn{2}{|c|}{ Input data } & \multicolumn{2}{c|}{$\begin{array}{c}\text { Target } \\
\text { Data }\end{array}$} \\
\hline 1 & 88906 & 1094 & 0 & 1 \\
\hline 2 & 89996 & 4 & 0 & 1 \\
\hline 3 & 89995 & 5 & 0 & 1 \\
\hline 4 & 89807 & 193 & 0 & 1 \\
\hline. &. &. &. &. \\
\hline. &. &. &. &. \\
\hline 46 & 89761 & 239 & 1 & 0 \\
\hline 47 & 89847 & 153 & 1 & 0 \\
\hline 48 & 89973 & 27 & 1 & 0 \\
\hline
\end{tabular}

In this work 48 samples of fingerprints is considered. For the sake of simplicity, only two class of fingerprints is considered i.e loops and whorls. The histogram values form the dataset for the feed-forward neural network for training. Table 1 depicts the sample data set.

\section{EXPERIMENTS AND RESULTS}

A two layer feed-forward back propagation neural network has been used for complete experiment. The network depicted in figure 9 used for the complete experiment.

Neural Network tool of Matlab 10 is used for the complete work. Three different kinds of samples are applied on the network to perform different activities like -

- Training: These are presented to the network during training and the network is adjusted according to its error.

- Validation: It is used to measure network generalization, and to halt training when generalization stops improving.

- Testing: It is used only for testing the final solution in order to confirm the actual predictive power of the network.

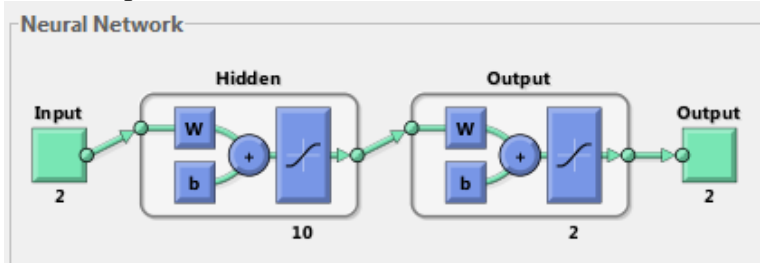

Figure 9: Feed Forward neural network with 10 hidden layers

The UI screen is made simple as shown in Figure 10.The user is expected to train the network using Train butto $n$ with the dataset statically embedded in the code. Next, the user chooses a sample fingerprint using Choose File button which gives a popup to select a fingerprint image. Eventually, the user presses the Test button which initiates the classification process. A pop up window displays the class of fingerprint, as well as the character exhibited by the possessor of such a fingerprint. 


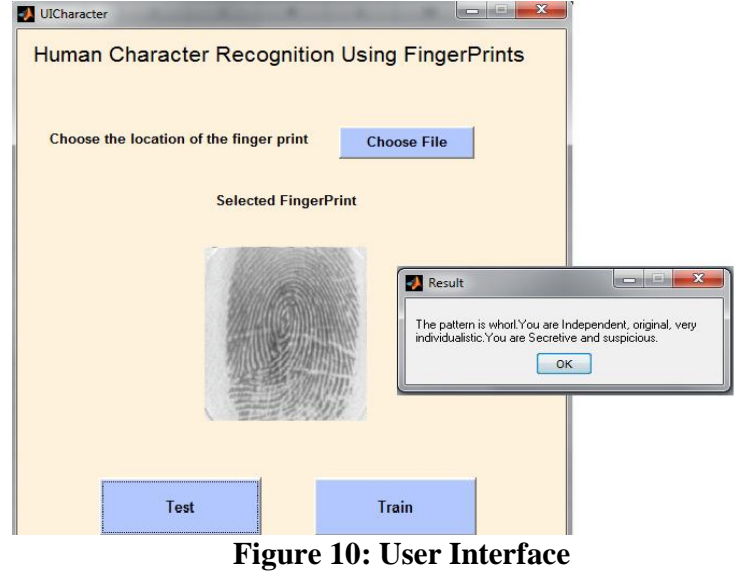

It has been found that more we train the classifier more accurate the classification result will be. In the experiment conducted, out of 10 fingerprints, 8 fingerprints were classified correctly giving an accuracy of $87.5 \%$ and the character exhibited by such a fingerprint matches almost $75 \%$.The confusion matrix depicted in Figure 11 supports the result.

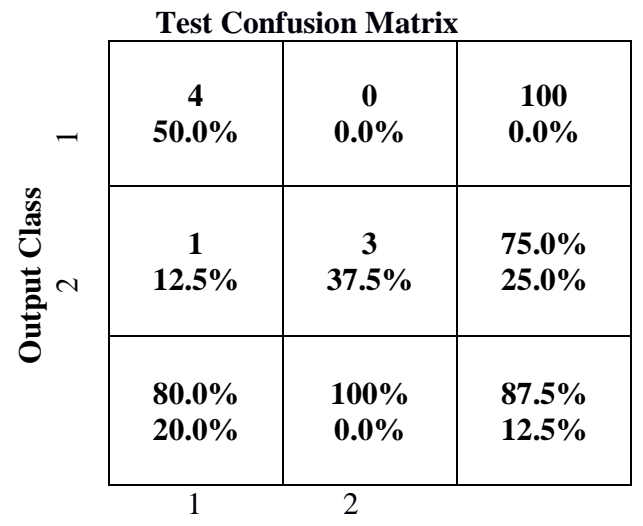

Target Class

Figure 11: Test Confusion Matrix

\section{APPLICATIONS}

Getting the measure of the character of a person is always considered advantageous before trusting him with any kind of work in any field. Only the subset of plethora of the applications are presented here-

Civil Service: In Civil services department, people with lot of courage, intelligence, diplomatic skills and ability to withstand lot of pressure are preferred over others. In such case we could consider people with lots of loops in their fingers.

Military Service: People in military services should be secretive and suspicious in nature. In such cases, people with whorl patterns are preferred.

Parents in estimating the talent of their wards: Cheiro, always wanted Palmistry should be read by parents with special care as they could measure the character of their children very early and encourage them towards better paths in their life. For eg- a child with tented arch finger print could be encouraged to indulge in field of arts.

\section{CONCLUSION}

The entire human body reflects the character of its possessor more accurately than any set of questionnaires fired at him. Among which, Palmistry holds the niche position in assessing personal traits as it is one of the oldest science and lot of study has been made in this field by many eminent people. With the aid of modern technology of biometrics, the day of having the computer to assess human character accurately is not far away.

In this work, fingerprints which don't change during the entire lifetime is considered as a tool to judge human character.

The artificial neural network method was employed to classify the fingerprint patterns into various categories. The experimental result showed that this could be done with $87.5 \%$ accuracy and character estimation could be done with $75 \%$ accuracy. As the number of samples are increased to train the classifier we could improve the classification accuracy of fingerprint patterns, eventually resulting in better estimation of human character.

\section{ACKNOWLEDGMENTS}

The authors are deeply indebted to the support provided by Department of Computer Science and Engineering, SJCE, Mysore during the course of this work. Heartfelt thanks are also due to Prof. Gangadhar Shastri of Maharaja Sanskrit Pathsala, Mysore for helping the authors to understand Palmistry from the vedic premise. Lastly, but not the least, the authors would like to thank students at MTechComputer Engineering,SJCE,2013 batch for always willing to extend their hand for their character analysis and bearing their sometimes inaccurate predictions.

\section{REFERENCES}

[1] [Web],"http://www.biometricsociety.org/about/definition -of- biometrics/."'

[2] [Web], "en.wikipedia.org/wiki/biometrics."

[3] C.N.Ravikumar,P Girish Chandra and Narayana R, "Future path way to biometric," International Journal of Biometrics and Bioinformatics (IJBB), vol. 5, no. 3, p. $180,2011$.

[4] [Web],"http://en.wikipedia.org/wiki/palmistry\#history.”

[5] W. G. Benham, The Laws of Scientific Hand Reading. 2013.

[6] J. R. Vacca, Biometric Technologies and Verification Systems. 2007.

[7] Siddhesh Angle, Reema Bhagtani and Hemali Chheda, "Biometrics : A further echelon of security"

[8] M. M. Hood, Triguna: A hindu-Balinese Philosphy for Gamelan Gong Gede Music. Transaction Publishers, 2010.

[9] A. C. N. Michael Kuckena, "Fingerprint formation," Journal of Theoretical Biology 235 (2005)71-83, 2005.

[10] [Web],"http://psychochirology.com/fingerprintsrevealed. html."

[11] [Web], "http://en.wikipedia.org/wiki/psychometrics." 
[12] A. Argelander, "The personal factor in judging human character," Journal of Personality, vol. 5, no. 4, pp. 285-295, 1937.

[13] [Web],"http://www.crystalinks.com/facialanalysis.htm"

[14] D. B. Dwivedi, Wonders of Palmistry. 2002.

[15] [Web], "http://linesandgems.com/palmistry.aspx."

[16] [Web], "http://en.wikipedia.org/wiki/fingerprint."

[17] [Web],"http://www.odec.ca/projects/2004/fren4j0/public html/fingerprint patterns.htm."

[18] F.B.I, The Science of Fingerprints: classification and uses. United States Dept. of Justice, Federal Bureau of Investigation, 1979.

[19] S.Fenton, Simply Series: Simply Palmistry. Sterling Publishing Company, Inc, 2005.

[20] K. Z. Jones, The Everything Palmistry Book: Discover What the Future Holds-Life, Love, and Wealth-All in the Palm of Your Hand. Adams Media, 2010.

[21] Jyoti Rajharia and P.C. Gupta, "A new and effective approach for fingerprint recognition by using feed forward back propagation neural network," International Journal of Computer Applications, vol. 52, August 2012. 